AIMS Genetics, 3(4): 205-211.

A I M S Genetics

DOI: $10.3934 /$ genet.2016.4.205

Received: 01 August 2016

Accepted: 27 September 2016

Published: 09 October 2016

http://www.aimspress.com/journal/Genetics

Case report

\title{
Changes in FGFR2 amino-acid residue Asn549 lead to Crouzon and Pfeiffer syndrome with hydrocephalus
}

\section{Caroline Apra $^{1,2}$, Corinne Collet ${ }^{3, *}$, Eric Arnaud ${ }^{1}$ and Federico Di Rocco ${ }^{4}$}

1 Department of Neurosurgery, Hôpital Necker-Enfants Malades, Paris, France-Centre de référence des dysostoses craniofaciales

2 Sorbonne Universités, Université Pierre et Marie Curie, Paris, France

3 Department of Biochemistry and Genetic Biology, Inserm 1132, Hôpital Lariboisière, Paris, France

4 Department of Pediatric Neurosurgery, Hôpital Neurologique Pierre Wertheimer, Lyon, France

* Correspondence: Email: corinne.collet@aphp.fr; Tel: +3314-995-6435; Fax: +3314-995-8477.

\begin{abstract}
Mutations in Fibroblast Growth Factor Receptor II (FGFR2) have been identified in patients with Crouzon and Pfeiffer syndrome, among which rare mutations of the intracellular tyrosine kinase domain. Correlating subtle phenotypes with each rare mutation is still in progress. In Necker-Enfants Malades Hospital, we identified three patients harboring three different pathogenic variants of the same amino acid residue Asn-549 located in this domain: in addition to a very typical crouzonoid appearance, they all developed clinically relevant hydrocephalus, which is an inconstant feature of Crouzon and Pfeiffer syndrome. Overall, FGFR2 tyrosine kinase domain mutations account for 5/67 (7.4\%) cases in our hospital. We describe a novel mutation, p.Asn549Ser, and new cases of p.Asn549His and p.Asn549Thr mutations, each reported once before. Our three cases of Asn-549 mutations, alongside with rare previously reported cases, show that these patients are at higher risk of hydrocephalus. Clinical and imaging follow-up, with possible early surgery, may help prevent secondary intellectual disability.
\end{abstract}

Keywords: Craniosynostosis; craniostenosis; FGFR2; craniofacial syndrome; hydrocephalus; cavum septum pellucidum; intellectual disability 


\section{Introduction}

Craniosynostosis is a cranial malformation occurring in approximately 1 in 2500 live births. In around $15 \%$ of these cases [1,2], it is part of a craniofacial syndrome associating typical facial features such as exorbitism and maxillary retrusion to the premature fusion of cranial sutures [3]. Common variable features also include secondary complications, such as hydrocephalus, intellectual disability, or sleep apnea. However, the diversity of phenotypes is not always straightforwardly explained by the genetic alteration. Knowing the phenotypical prognosis helps managing and preventing some complications, by proposing early adapted treatment.

FGFR2 (Fibroblast Growth Factor Receptor 2) is a key gene involved in craniosynostosis syndromes such as Crouzon and Pfeiffer syndromes. It codes for a membrane receptor with three extracellular immunoglobulin(Ig)-like domains, a transmembrane domain and an intracellular tyrosine kinase (TK) domain. It is expressed from gastrulation onwards in the developing skeleton, particularly in the frontal bones of the skull and in the limbs [4].

Since 1994, many FGFR2 mutations have been identified in patients with Crouzon syndromes [4-8]. Most of them are missense mutations affecting the extracellular domain $[9,10]$. Besides, rare mutations affect the intracellular TK domain of FGFR2 with subsequent constitutive activation [9,11]. The clinical phenotypes associated with these rare mutations have been described but there is little genotype-phenotype correlation regarding subtle findings. Therefore, all cases must still be centralized and described precisely.

From a cohort of patients affected by craniofacial syndromes, we identified three patients with missense mutations affecting the same amino acid Asn549, located in the FGFR2 TK domain, we discuss their molecular mechanism and compare their phenotypes.

\section{Materials and Methods}

Blood samples were drawn for diagnostic purposes after informed consent. DNA was extracted using a QIAamp blood kit (Qiagen, Courtaboeuf, France). FGFR2 exons 4, 6, 8 (IIIa), 10 (IIIc), 11, 14, 15, 16, 17 were amplified and sequenced directly on both strands (Life technologies, Courtaboeuf, France) then analyzed using Sescape (Life Technologies and Alamut software (Interactive Biosoftware, Rouen, France). The NCBI reference sequences were FGFR2 (NM_000141.4) for c.DNA and FGFR2 (NG_012449.1) for exons numbering. The mutations nomenclature is based on HGVS nomenclature guidelines (http://www.hgvs.org/mutnomen).

\section{Case reports}

Patient $\mathbf{1}$ is a sixteen-year-old boy with Crouzon syndrome, showing facial retrusion and prominent forehead. His parents and four siblings show no clinical sign of craniosynostosis. Pregnancy was uneventful, head circumference at birth was $33 \mathrm{~cm}$. He was born with isolated sagittal suture fusion. Hydrocephalus at birth required ventriculo-peritoneal shunt, that was removed at the age of ten, after decompression surgery. Other surgical history includes forehead advancement at one, and extensive frontal surgery at three, followed by frontal subcutaneous fat injection. He never presented papilledema. Recent brain imaging reveals asymptomatic cavum septum pellucidum, 
but no ventricular enlargement nor Chiari malformation, which is a displacement of cerebellar tonsils through the foramen magnum, sometimes associated with craniostenosis (Figure 1a). Recent clinical evaluation reveals frequent headaches, behavioral difficulties and intellectual disability (IQ 70), with normal neurological and sensory examination.

This patient presented c.1646A $>\mathrm{G}$, p.Asn549Ser mutation, which has been reported in the Human Gene Mutation Database (Cardiff University, 2015) but has never been described clinically (Figure 2).

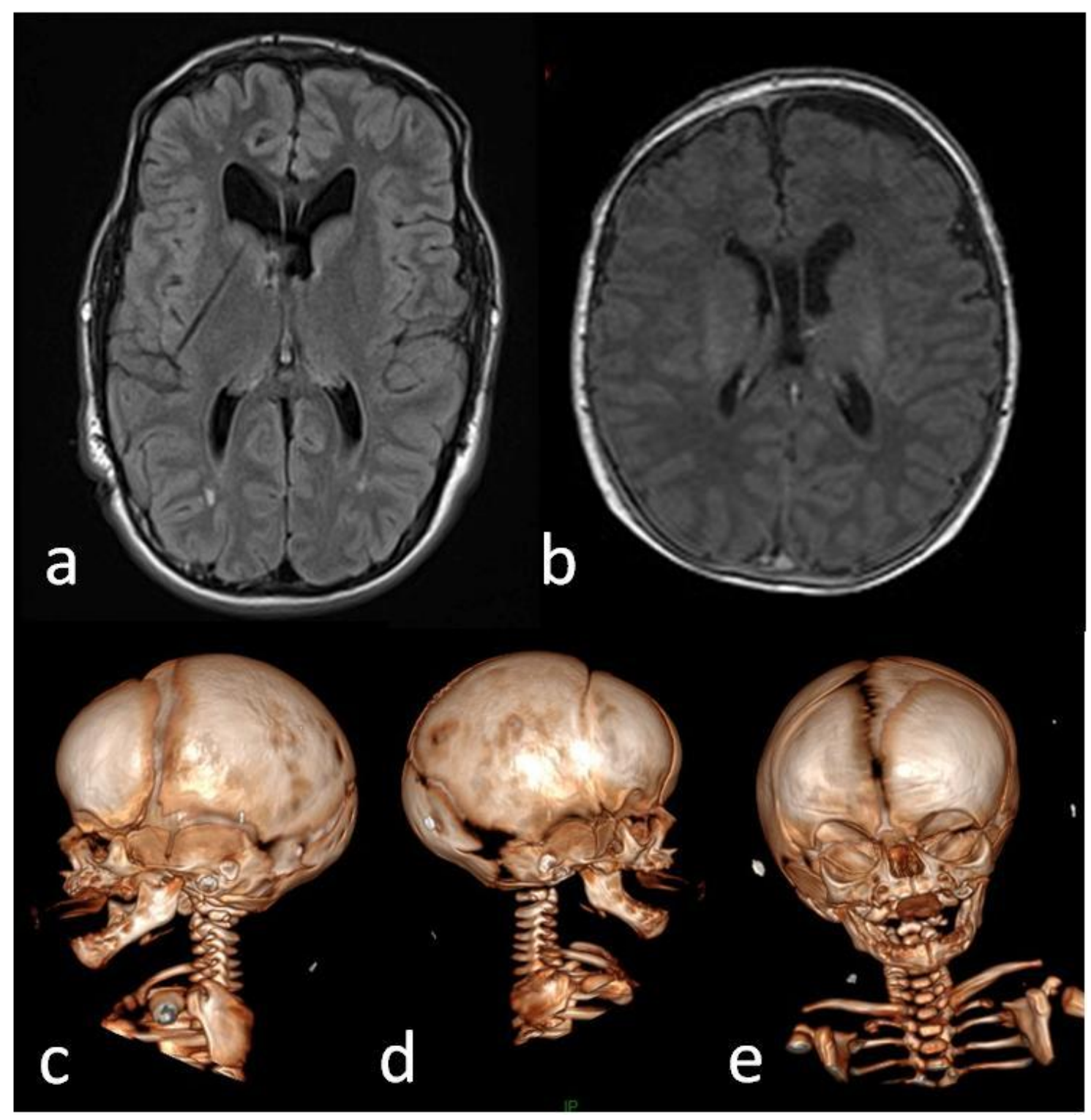

Figure 1. a) Case 1 at the age of 14, brain MRI (T1 sequence, axial) shows cavum septum pellucidum and the sequelae of a right ventricular catheter, as hydrocephalus had been treated with ventriculo-peritoneal shunting; b) c) d) e) Case 3 at the age of 1 before decompressive craniectomy was performed: b) Brain MRI (T1 sequence, axial) shows cavum septum pellucidum and mild ventricular dilatation. c) d) e) 3D CT scan shows right coronal suture fusion and lambdoid ridge. 


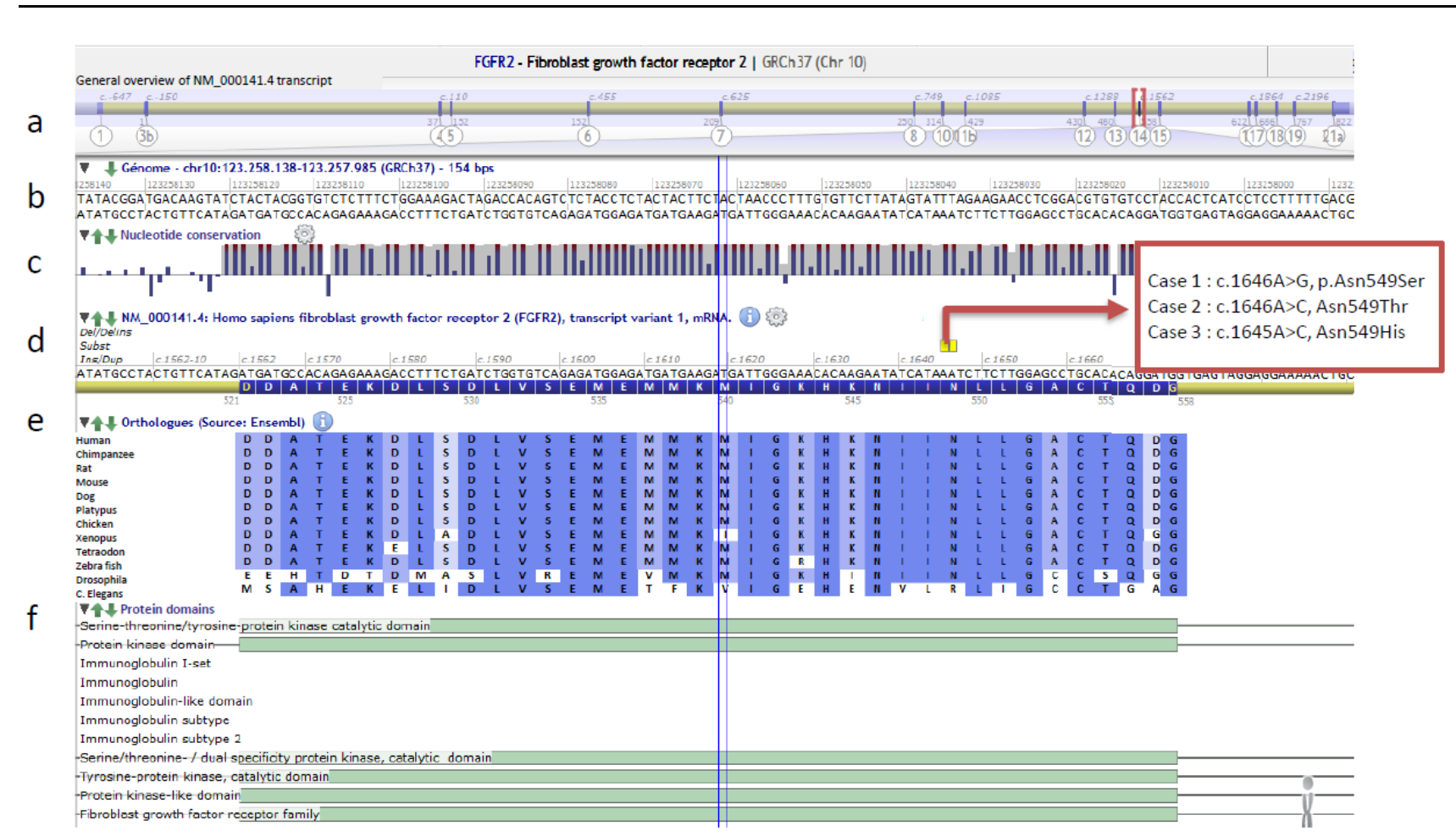

Figure 2. Localization of mutations in FGFR2 exon 14, coding for FGFR2 tyrosine kinase 1 domain (Alamut Visual Interactive Biosoftware, France). a) general overview of Humo Sapiens FGFR2 (NM_000141.4) transcript, composed of 18 exons with genomic numbering, exon 14 is shown in red brackets; b) exon 14 genomic localization; c) nucleotide conservation; d) amino-acid positions: the mutations localizations for the three cases are shown in yellow (red arrow); e) amino-acid conservation among different species: the mutations are located in highly conserved nucleotides; f) exon 14 transcript, this exon codes for tyrosine kinase domain.

Patient 2 is a fifteen-year-old girl presenting with exorbitism, maxillary retrusion, and broad thumbs that corresponds to Pfeiffer syndrome. Parents have a normal appearance and further family history is unknown. Pregnancy was uneventful. She was born with coronal fusion, then developed premature sagittal fusion. Ventriculoperitoneal shunt was placed at the age of six weeks for hydrocephalus, and was removed at ten. She underwent bilateral parietal decompression at the age of four and ten, posterior decompression with distractors at thirteen. Ophthalmological examination revealed strabismus, which was treated surgically, and right ptosis. Neurological imaging shows Chiari malformation, which was operated at the age of five, complicated with cervical C3-C7 syrinx, and no intracranial malformation. Clinical examination shows unilateral hypoacousia, upper limbs paresthesias and lower limbs weakness that progressively got better after occipital foramen surgery. She presents behavioral abnormalities treated with ritaline but no major intellectual disability. She has sleep apnea syndrome, which required CPAP therapy at night for years and resolved after tonsillectomy.

She was diagnosed with c.1646A>C, Asn549Thr mutation, reported once [12] (Figure 2). 
Patient 3 is a four-year-old boy presenting with typical Crouzon syndrome with exorbitism and maxillary retrusion, associated with right coronal suture fusion and lambdoid ridge at birth (Figure 1c, d, e). His father and father's mother had a crouzonoid appearance but were not followed. Pregnancy was uneventful, head circumference at birth was $38.5 \mathrm{~cm}$. He underwent fronto-orbital advancement with decompressive craniectomy at the age of one, tonsillectomy at two. At the age of three, he presented premature bilateral coronal and sagittal fusion, for which he underwent parietal decompression. Ophthalmological examination at the time revealed bilateral papilledema, a sign of hydrocephalus, that resolved after surgery, and left ptosis. Recent head CT scan shows asymptomatic cavum septum pellucidum, no ventricular enlargement nor cerebellar tonsils malformation (Figure 1b). There is no neurological or sensory impairment, and no major intellectual disability. He developed moderate sleep apnea syndrome (apnea+hypopnea index $0.8 / \mathrm{min}$ ).

He presented with c.1645A $>$ C, Asn549His mutation, which has been described already [9] (Figure 2).

\section{Discussion}

Mutations affecting FGFR2 TK domain are a subgroup of FGFR2 mutations found in syndromic craniosynostoses. They may correlate with a phenotypical subgroup, with particular characteristics and complications.

Although most FGFR2 mutations found in patients with Crouzon and Pfeiffer syndromes are located in exons 8 (IIIa) and 10 (IIIc) that encode the extracellular part of the protein $[9,10]$, screening of patients with syndromic craniosynostosis has led to the identification of nine distinct mutations in FGFR2 intracellular TK domain (exons 14, 15, 16), accounting for $\sim 10 \%$ of patients with craniosynostosis [9,11-14]. In Necker-Enfants Malades Hospital, screening of FGFR2 in all patients with Crouzon and Pfeiffer syndromes led to the identification of $7.4 \%$ of TK domain mutations (5/67), 4 affecting Asn549 and one Lys659. Patients with other syndromic craniosynostoses did not present with TK domain mutations. Only three patients with Asn549 variants were available for complete clinical follow-up. The three mutations are localized in c.1645A and c.1646A, highly conserved nucleotides, which is consistent with their pathological impact (Figure 2). When screening FGFR2 for all patients with any syndromic craniosynostosis, Kan et al. found 6 mutations $(7 \%, 6 / 85)$ occurring in the TK domain, in 8 patients $(3 \%, 8 / 259)$ [9] which is consistent with our population. On the opposite, Roscioli et al. do not report any mutation in the TK domain in a review of 630 patients, but it is likely that they have not been screened for this domain [15].

The protein structure itself is not affected by the Asn549 changes, according to simulated Polyphen scores (score $\max =1$ for both SIFT and Polyphen softwares). The mechanism by which these mutations lead to FGFR2 gain of function has been explored by Chen et al., who compared the crystal structures of wild-type FGFR2 kinase domain with those of N549H and N549T [11]. They found that these mutations lead to ligand-independent FGFR2 kinase activation by directly disengaging a «molecular brake» that is conserved in a wide range of receptors tyrosine kinase. More precisely, N549 maps onto the kinase hinge (the linker between $\beta 5$ strand and $\alpha \mathrm{D}$ helix). Crystal analysis of the hinge region of the $\mathrm{N} 549 \mathrm{H}$ and N549T mutant kinases reveals the loss of the hydrogen bonds between mutated residue 549 and the backbone atoms of the loop between the $\alpha \mathrm{C}$ helix and the $\beta 4$ strand, relaxing the N-lobe to rotate towards the C-lobe. This mechanism prevents 
normal function of this molecular break and constitutively activates FGFR2 for p.Asn549His and p.Asn549Thr changes. We can hypothesize that the Asn549Ser mutation has the same effect.

On a phenotypical point of view, the three patients we report present with typical Crouzon or Pfeiffer syndrome: exorbitism, maxillary retrusion, premature suture fusion, and one patient with hand abnormalities. However, the three of them developed hydrocephalus, which happens in only $40 \%$ of patients with Crouzon and Pfeiffer syndrome in general [16]. Two patients have a cavum septum pellucidum, which is found in only $1.1 \%$ of general population. Among patients with syndromic craniosynostosis, cavum septum pellucidum has been reported in $25 \%$ of patients with Apert syndrome, a congenital disorder characterized by craniosynostosis associated with malformations of the hands and feet [17], but not in Crouzon patients. This finding was considered incidental, but may also be interpreted as obstructing cerebrospinal fluid circulation, and thus causing hydrocephalus. Hydrocephalus may cause intellectual disability, which could explain this tendency in our three patients, and in the cases reported by Wilkie et al. and Kan et al. [9,12].

The two patients with p.Asn549His mutations reported by Kan et al. display Crouzon appearance with developmental delay and hydrocephalus, and one of them has craniosynostosis with Chiari malformation, while the other has macrocephaly [9]. The newly reported p.Asn549Ser mutation is also associated, in our case, with hydrocephalus and secondary intellectual disability.

Overall, although no definitive conclusion can be drawn from so few cases, hydrocephalus seems to be an atypically frequent feature in patients with mutations in the FGFR2 TK domain: patients with p.Lys526Glu mutation display scaphocephaly with intellectual disability [18]. Kan et al. already reported that hydrocephalus and intellectual disability were over-represented in patients with TK domains mutations compared with typical Crouzon and Pfeiffer syndromes [9].

\section{Conclusion}

Patients with Crouzon and Pfeiffer syndromes presenting with missense mutations of Asn549 residue, located in FGFR2 TK domain, are at high risk of developing hydrocephalus. Therefore, regular imaging and clinical monitoring is useful to treat hydrocephalus as early as possible and prevent secondary intellectual disability.

\section{Conflict of interest}

All authors declare no conflict of interest in this paper.

\section{References}

1. Agochukwu NB, Solomon BD, Muenke M (2012) Impact of genetics on the diagnosis and clinical management of syndromic craniosynostoses. Childs Nerv Syst 28: 1447-1463.

2. Rocco FD, Arnaud E, Renier D (2009) Evolution in the frequency of nonsyndromic craniosynostosis. J Neurosurg Pediat 4: 21-25.

3. Crouzon O (1912) Dysostose cranio-faciale hereditaire. Bull Mem Soc Med Hop Paris 33: 545-555.

4. Reardon W, Winter RM, Rutland P, et al. (1994) Mutations in the fibroblast growth factor receptor 2 gene cause Crouzon syndrome. Nature Genetics 8: 98-103. 
5. Collet C, Alessandri JL, Arnaud E, et al. (2014) Crouzon syndrome and Bent bone dysplasia associated with mutations at the same Tyr-381 residue in FGFR2 gene. Clin Genet 85: 598-599.

6. Lajeunie E, Heuertz S, Ghouzzi VE, et al. (2006) Mutation screening in patients with syndromic craniosynostoses indicates that a limited number of recurrent FGFR2 mutations accounts for severe forms of Pfeiffer syndrome. Eur J Hum Genet 14: 289-298.

7. Lajeunie E, Ma HW, Bonaventure J, et al. (1995) FGFR2 mutations in Pfeiffer syndrome. Nat Genet 9: 108.

8. Tartaglia M, Di Rocco C, Lajeunie E, et al. (1997) Jackson-Weiss syndrome: identification of two novel FGFR2 missense mutations shared with Crouzon and Pfeiffer craniosynostotic disorders. Hum Genet 101: 47-50.

9. Kan S, Elanko N, Johnson D, et al. (2002) Genomic screening of fibroblast growth-factor receptor 2 reveals a wide spectrum of mutations in patients with syndromic craniosynostosis. Am J Hum Genet 70: 472-486.

10. Meyers GA, Day D, Goldberg R, et al. (1996) FGFR2 exon IIIa and IIIc mutations in Crouzon, Jackson-Weiss, and Pfeiffer syndromes: evidence for missense changes, insertions, and a deletion due to alternative RNA splicing. Am J Hum Genet 58: 491-498.

11. Chen H, Ma J, Li W, et al. (2007) A molecular brake in the kinase hinge region regulates the activity of receptor tyrosine kinases. Mol Cell 27: 717-730.

12. Wilkie AOM, Bochukova EG, Hansen RMS, et al. (2007) Clinical dividends from the molecular genetic diagnosis of craniosynostosis. Am J Med Genet 143: 1941-1949.

13. de Ravel TJL, Taylor IB, Van Oostveldt AJT, et al. (2005) A further mutation of the FGFR2 tyrosine kinase domain in mild Crouzon syndrome. Eur J Hum Genet 13: 503-505.

14. Zankl A, Jaeger G, Bonafé L, et al. (2004) Novel mutation in the tyrosine kinase domain of FGFR2 in a patient with Pfeiffer syndrome. Am J Med Genet A 131: 299-300.

15. Roscioli T, Elakis G, Cox TC, et al. (2013) Genotype and clinical care correlations in craniosynostosis: findings from a cohort of 630 Australian and New Zealand patients. Am J Med Genet C Semin Med Genet 163C: 259-270.

16. Collmann H, Sörensen N, Krauss J (2005) Hydrocephalus in craniosynostosis: a review. Childs Nerv Syst 21: 902-912.

17. Raybaud C, Di Rocco C (2007) Brain malformation in syndromic craniosynostoses, a primary disorder of white matter: a review. Childs Nerv Syst 23: 1379-1388.

18. McGillivray G, Savarirayan R, Cox T, et al. (2005) Familial scaphocephaly syndrome caused by a novel mutation in the FGFR2 tyrosine kinase domain. J Med Genet 42: 656-662.

(C) 2016 Caroline Apra et al., licensee AIMS Press. This is an open access article distributed under the terms of the Creative Commons Attribution License

(http://creativecommons.org/licenses/by/4.0) 